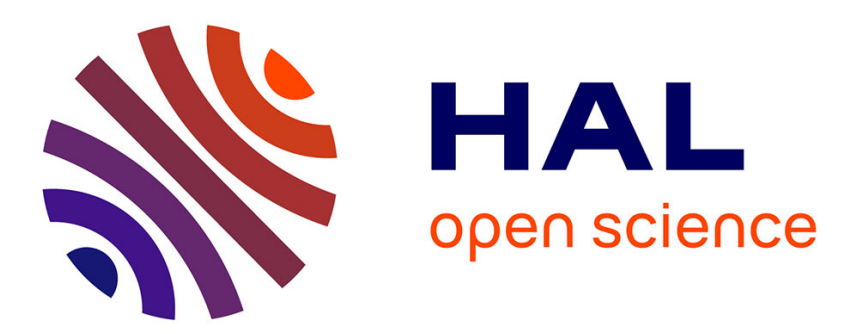

\title{
Hybrid Eulerian-Lagrangian method for soot modelling applied to ethylene-air premixed flames
}

Nicolas Dellinger, Nicolas Bertier, Francis Dupoirieux, Guillaume Legros

\section{To cite this version:}

Nicolas Dellinger, Nicolas Bertier, Francis Dupoirieux, Guillaume Legros. Hybrid Eulerian-Lagrangian method for soot modelling applied to ethylene-air premixed flames. Energy, 2020, 194, 116858, p. 1-10. 10.1016/j.energy.2019.116858 . hal-02456130

\section{HAL Id: hal-02456130 \\ https://hal.science/hal-02456130}

Submitted on 27 Jan 2020

HAL is a multi-disciplinary open access archive for the deposit and dissemination of scientific research documents, whether they are published or not. The documents may come from teaching and research institutions in France or abroad, or from public or private research centers.
L'archive ouverte pluridisciplinaire HAL, est destinée au dépôt et à la diffusion de documents scientifiques de niveau recherche, publiés ou non, émanant des établissements d'enseignement et de recherche français ou étrangers, des laboratoires publics ou privés. 


\section{Journal Pre-proof}

Hybrid Eulerian-Lagrangian method for soot modelling applied to ethylene-air premixed flames

Nicolas Dellinger, Nicolas Bertier, Francis Dupoirieux, Guillaume Legros

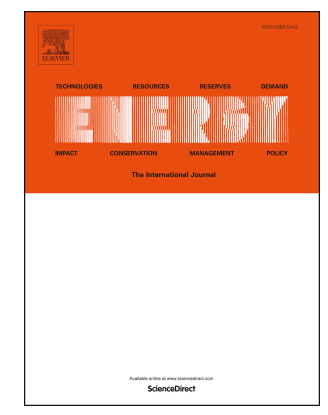

PII:

S0360-5442(19)32553-8

DOI:

https://doi.org/10.1016/j.energy.2019.116858

Reference: EGY 116858

To appear in: Energy

Received Date: 17 July 2019

Revised Date: 18 December 2019

Accepted Date: 24 December 2019

Please cite this article as: Dellinger N, Bertier N, Dupoirieux F, Legros G, Hybrid Eulerian-Lagrangian method for soot modelling applied to ethylene-air premixed flames, Energy (2020), doi: https:// doi.org/10.1016/j.energy.2019.116858.

This is a PDF file of an article that has undergone enhancements after acceptance, such as the addition of a cover page and metadata, and formatting for readability, but it is not yet the definitive version of record. This version will undergo additional copyediting, typesetting and review before it is published in its final form, but we are providing this version to give early visibility of the article. Please note that, during the production process, errors may be discovered which could affect the content, and all legal disclaimers that apply to the journal pertain.

(C) 2019 Published by Elsevier Ltd. 


\section{Highlights}

\section{Hybrid Eulerian-Lagrangian Method For Soot Modelling Applied To Ethylene-Air Premixed} Flames

Nicolas Dellinger, Nicolas Bertier, Francis Dupoirieux, Guillaume Legros

- Soot particles dynamics is described through a detailed Lagrangian tracking method.

- Soot particles growth and oxidation processes are taken into account.

- Soot particles interactions are limited with success to coalescence as a first guess.

- Particle size distributions are extracted efficiently through population reduction.

- Targeted flames for validation are laminar premixed ethylene-air flames. 


\title{
Hybrid Eulerian-Lagrangian Method For Soot Modelling Applied To Ethylene-Air Premixed Flames
}

\author{
Nicolas Dellinger ${ }^{\mathrm{a}, *}$, Nicolas Bertier $^{\mathrm{b}}$, Francis Dupoirieux $^{\mathrm{c}}$, Guillaume Legros $^{\mathrm{d}}$ \\ ${ }^{a}$ Department MultiPhysics for Energetics (DMPE), ONERA, Université Paris Saclay, F-92322 Châtillon, France \\ ${ }^{b}$ Department MultiPhysics for Energetics (DMPE), ONERA, Université Paris Saclay, F-92322 Châtillon, France \\ ${ }^{c}$ Scientific Directorate, ONERA, Université Paris Saclay, F-91123 Palaiseau, France \\ ${ }^{d}$ Sorbonne Université, Centre National de la Recherche Scientifique, UMR 7190, Institut Jean le Rond 2'Alembert, F-75005 \\ Paris, France
}

\begin{abstract}
Soot formation has become an important issue in the design of gas turbine combustors due to its environmental impact and its contribution to radiative heat transfer in the combustion chamber. However, efficient and accurate prediction of soot particles formation, growth, oxidation and interaction in gas turbine combustors is still an open field in computational fluid dynamics. The present approach proposes to combine a reduced gas-phase chemistry, a sectional model for polycyclic aromatic hydrocarbons, and a Lagrangian description of soot particles dynamics. The Lagrangian description has been chosen for its ability to simulate the evolution of the particle size distribution. A numerical procedure is proposed to minimise its CPU cost. This approach was successfully applied to the simulation of steady laminar premixed ethylene-air flames at three fuel equivalence ratios, which constitutes a prerequisite towards its use in an aeronautical combustion chamber.
\end{abstract}

Keywords: CFD, Combustion, PAH, Sectional model, Soot, Lagrangian description

\section{Introduction}

Soot particles created in gas turbine combustors contribute to local air pollution, particularly in airport areas, and enhance the radiative transfer in the combustion chamber, increasing the gas temperature and the thermal load on the chamber walls and turbine blades. The production of other pollutants, such as nitrogen oxides which are particularly sensitive to temperature, might be influenced too [1]. As a consequence of both concerns, efficient and accurate modelling of soot formation and evolution is an important issue in the design of aircraft engines, but remains an open field due to the complexity of the phenomena involved. The chemical kinetics of gaseous soot precursors formation from the fuel, as well as of soot particles formation from the precursors, has in particular to be described. The interactions of the soot particles with the surrounding gas, leading to their growth or oxidation, as well as the interactions between the particles must also be considered.

Soot models are generally classified in three categories. Empirical models use correlations from experimental measurements to compute the soot volume fraction and number density, for instance as a function of the fuel

\footnotetext{
* Corresponding author

Email address: nicolas.dellinger@onera.fr (Nicolas Dellinger)
} 
concentration [2], the concentration of an intermediate species [3], or a location in the flame [4]. Suitable with global reaction schemes, and thus allowing the calculation of complex configurations, these models are however weakly predictive over wide ranges of parameters.

Semi-empirical models, as the Leung one [5], are based on a simplified description of soot nucleation, coagulation, growth, and oxidation kinetics. Source terms for the soot volume fraction and number density are calculated from kinetic constants, determined from experimental measurements, or derived from a population balance equation for coagulation. More predictive, these models generally require the concentration of acetylene, as light soot precursor, computed by means of expensive reaction mechanisms or cheaper tabulated chemistry [6]. Application of semi-empirical models to the simulation of industrial burners is therefore usually based on the tabulation approach.

Detailed models, of particular interest for the present study, include a complex description of both soot chemical kinetics and particles dynamics. Reactions for surface growth by addition of acetylene and condensation of polycyclic aromatic hydrocarbons (PAHs), as well as oxidation by $\mathrm{O}_{2}$ and $\mathrm{OH}$ are generally included in the reaction mechanism [7]. Following the requirement to correctly predict these species and accurately describe soot nucleation, a large number of intermediate species, from benzene up to large PAHs, has to be added to the kinetic scheme, resulting in mechanisms of tens of species and hundreds of reactions [8]. Particles dynamics, which includes soot particles evolution via the aforementionned chemical processes associated with coalescence and aggregation, can be modelled using sectional methods [9, 10, 11] or methods of moments $[12,13]$. Unlike empirical and semi-empirical models, the two approaches account for both the polydispersion in size and the non-sphericity of the particles, which can be described as fractal aggregates $[10,14,15,16,17]$. Methods of moments, for which a restricted set of moments can be sufficient to characterize the soot particles population, are yet preferable to limit the CPU cost in industrial applications, sectionals methods requiring a high number of sections and, eventually, variables per section. Because of the large chemical mechanism required to predict the evolution of the soot precursors and the need to properly describe the particles dynamics, detailed soot models can be prohibitely expensive for the computation of aircraft engines combustion chambers. Tabulated chemistry $[18,19,20]$ might be a solution to limit the number of transported variables relative to the fuel combustion. However, the modelling of heat losses as well as the consideration of combustion regimes and multiphase flow effects increase the complexity and thus the computational cost of such approaches. Also, the extension of tabulated chemistry to species having long evolution time scales, such as NO or soot, is an issue [21]. Alternatively, sectional methods can be used to characterize PAH growth [10, 22, 23] and combined with a reduced chemical mechanism for lighter species $[24,25]$. The present study follows the latter strategy and focuses on the Lagrangian description of soot particles as a dispersed solid phase. The Lagrangian tracking method is a substitute to sectional methods and methods of moments for computing particle dynamics. It gives the possibility to follow a numerical particle continuously along its trajectory, and to monitor its individual interaction with both the gas phase and the other particles. No classes or sections of particles have here to be prescribed, and the calculation of the particle size distribution, of first interest for soot applications, is straightforward. Nevertheless, the computational cost could be a limiting factor, the statistical convergence of a Lagrangian method being inversely proportional to the square root of the number of particles.

The Lagrangian tracking approach has been recently proposed by Gallen et al. [26] and Ong et al. [27]. While acetylene is used in these two studies, the soot particles are nucleated from PAH molecules and radicals in 
the present one. More detailed soot evolution models, which allow the two-way coupling of the gaseous and solid phases, are additionally used. An innovative algorithm to control the number of tracked particles is finally introduced in order to keep the computational cost moderate.

The model was implemented in the aerothermochemistry CEDRE code, developed at ONERA [28]. The approach being unusual compared to more common Eulerian methods, the subsequent simulations must be validated by comparison with academic flame configurations. While numerical simulations of steady twodimensional laminar sooting flames have already been conducted with some success [29], the discrimination between failures of the soot model and of the flow description that may be responsible for the discrepancies between experimental measurements and numerical fields especially remains an issue. For that reason, the present study is specifically focused on the validation based on steady one-dimensional laminar ethylene-air flames at three fuel equivalence ratios.

\section{Gas phase models and methods}

Through the coupling of dedicated solvers, the CEDRE code allows the simulation of multiphase compressible reacting flows on unstructured meshes. In particular, the conservation equations for the reacting gas phase in the present study are resolved through the finite-volume Eulerian solver CHARME, able to deal with future targeted applications to industrial configurations.

\subsection{Conservation equations}

For compressible laminar reacting flows, the mass, momentum, and energy conservation equations write:

$$
\begin{gathered}
\frac{\partial \rho Y_{k}}{\partial t}+\nabla \cdot\left(\rho Y_{k} \mathbf{V}\right)=-\nabla \cdot \mathbf{J}_{k}+\dot{\omega}_{k}+S_{k}^{d}, \\
\frac{\partial \rho \mathbf{V}}{\partial t}+\nabla \cdot(\rho \mathbf{V} \otimes \mathbf{V})=\nabla \cdot(\boldsymbol{\tau}-p \mathbf{I})+\rho \mathbf{g}+\mathbf{S}_{q}^{d}, \\
\frac{\partial \rho e_{t}}{\partial t}+\nabla \cdot\left(\rho e_{t} \mathbf{V}\right)=-\nabla \cdot \mathbf{J}_{e_{t}}+\nabla \cdot[(\boldsymbol{\tau}-p \mathbf{I}) \mathbf{V}]+\rho \mathbf{g} \cdot \mathbf{V}+S_{e_{t}}^{d}+S_{e_{t}}^{e x p}
\end{gathered}
$$

Momentum

where $t$ is the time, $\rho$ the density of the ideal gas mixture, composed of $N_{s}$ species, $Y_{k}$ the mass fraction of species $k, p$ the pressure, $\mathbf{V}$ the velocity vector, $\mathbf{g}$ the gravity vector, and $\mathbf{I}$ the identity matrix.

\section{Mass conservation equation (1)}

$\mathbf{J}_{k}$ is the molecular diffusion flux of species $k$, calculated using Hirschfelder and Curtiss' approximation [30] as:

$$
\mathbf{J}_{k}=\rho \mathcal{V}_{k} Y_{k}=-\rho\left(D_{k} \frac{\mathcal{M}_{k}}{\mathcal{M}} \nabla X_{k}+\mathbf{V}_{c} Y_{k}\right)
$$

where $\mathcal{V}_{k}, \mathcal{M}_{k}$ and $X_{k}$ are respectively the diffusion velocity, the molecular weight and the mole fraction of species $k$, and $\mathcal{M}$ the molecular weight of the mixture. $D_{k}$ is the diffusion coefficient of species $k$ in the mixture, computed by equation (5) as a function of the binary diffusion coefficients $\mathcal{D}_{j k}$ of each species $j$ in species $k$.

$$
D_{k}=\left(1-Y_{k}\right)\left(\sum_{j \neq k} \frac{X_{j}}{\mathcal{D}_{j k}}\right)^{-1}
$$


$\mathbf{V}_{c}$ is a correction velocity, given by equation (6), ensuring global mass conservation.

$$
\mathbf{V}_{c}=-\sum_{k=1}^{N_{s}} D_{k} \frac{\mathcal{M}_{k}}{\mathcal{M}} \nabla X_{k}
$$

$\dot{\omega}_{k}$ is the chemical source term of species $k$, calculated from the reaction mechanism [31]. $S_{k}^{d}$, the feedback source term of the dispersed phase - i.e. the soot particles - on species $k$, will be discussed in Subsection 4.3.

\section{Momentum conservation equation (2)}

$\tau$ is the viscous tensor, calculated for Newtonian fluids considered in the present study as:

$$
\boldsymbol{\tau}=\mu\left[\nabla \mathbf{V}+(\nabla \mathbf{V})^{T}\right]+\left(\kappa-\frac{2}{3} \mu\right)(\nabla \cdot \mathbf{V}) \mathbf{I},
$$

where $\mu$ is the dynamic viscosity of the mixture, defined as the arithmetic mean of the species viscosities $\mu_{k}$ weighted by the species mass fractions, and $\kappa$ the bulk viscosity, commonly assumed to be negligible. $\mathbf{S}_{q}^{d}$, the feedback source term of the dispersed phase, will be discussed in Section 3.

\section{Energy conservation equation (3)}

The energy conservation equation is written using the total energy, defined by equation (8), where $T$ is the temperature, $T_{0}$ the reference temperature, $h_{f_{k}}^{0}$ the specific enthalpy of formation of species $k$ at $T_{0}, c_{v}$ the specific heat capacity at constant volume of the mixture and $\mathcal{R}$ the gas constant:

$$
e_{t}=\sum_{k=1}^{N_{s}} Y_{k} h_{f_{k}}^{0}+\int_{T_{0}}^{T} c_{v} d T-\frac{\mathcal{R} T_{0}}{\mathcal{M}}+\frac{1}{2} \mathbf{V} \cdot \mathbf{V}
$$

$\mathbf{J}_{e_{t}}$ is the heat diffusion flux, computed under the assumption of negligible Dufour effect as:

$$
\mathbf{J}_{e_{t}}=-\lambda \nabla T+\sum_{k=1}^{N_{s}} h_{f_{k}}^{0} \mathbf{J}_{k}
$$

where $\lambda$ is the thermal conductivity of the mixture. $\lambda / \mu$ is defined as the arithmetic mean of the ratios $\lambda_{k} / \mu_{k}$ weighted by the species mass fractions, with $\lambda_{k}$ the thermal conductivity of species $k$.

$S_{e_{t}}^{d}$, the feedback source term of the dispersed phase, will be discussed in Section 3. Radiative heat transfers are not taken into account in equation (3), because, in the validation cases hereinafter, experimental temperature profiles are imposed through the corrective source term $S_{e_{t}}^{\exp }[6,32]$. It allows to make the calculation of the targeted burner-stabilised flames easier.

\subsection{Numerical methods}

A second order MUSCL (Monotonic Upwind Scheme for Conservation Laws) type scheme is used for space discretisation and combined with a HLLC (Harten-Lax-van Leer-Contact) scheme for hyperbolic fluxes. A second order Gear implicit scheme associated with a GMRES method for the resolution of the linear system is applied for time integration.

\section{Lagrangian dispersed phase solver}

Soot particles dynamics is described in the present study through the newly developed Lagrangian module SPARTACCUS of the CEDRE code. 


\subsection{Preliminary hypothesis}

Soot particles, whose diameter is in the range $1-80 \mathrm{~nm}$ hereinafter, are supposed to behave as tracers embedded in the gas flow. Their velocity is therefore taken equal to the gas velocity and their temperature, assumed uniform, to the gas temperature. Soot particles are furthermore modelled as solid spheres, with a constant density $\rho_{s}$.

Two collision regimes should be considered, coalescence and aggregation, depending on the age of the particles. Young soot particles, considered as liquid-like spheres [33], are indeed deformable and will preferably coalesce with their collision partner to form a larger spherical particle. Older carbonised particles, on the contrary, are the elementary solid bricks for the building of soot aggregates. The description of the latter is however not of primary interest for the comparison of numerical simulations and experimental measurements in the present study. Also computationally ${ }^{1}$ challenging, it will have to be addressed separately in the future. Aggregation is here replaced by "non-coalescence" to bound coalescence within its physical domain. To this end, the ageing of the soot particles is assumed to depend on a single parameter, their diameter, and a critical diameter $d_{\text {crit }}$ is introduced. Coalescence only occurs if one of the collision partners has a diameter smaller than $d_{c r i t}$. If not, "non-coalescence" takes place and the kinematic parameters of the collisions partners are equalised by analogy to aggregation.

The present formulation allows to directly compare the particle diameter to the primary particle diameter measured by $\mathrm{Xu}$ et al. [32] as part of the validation process in Section 5. Comparison of the soot volume fraction - the quantity usually available for complex configurations - is also straightforward, since not modified through aggregation at constant volume, at least as a first-order approximation. For the same reason, the soot radiative heat transfer might additionally be modelled as a function of the volume fraction [6]. Note that, in a flame with a mean temperature of $1500 \mathrm{~K}$ as in the following, the peak wavelength $\widehat{\lambda}$ involved in the radiative heat transfer would be of the order of $2 \mu \mathrm{m}$. This means that the size parameter $\pi d / \widehat{\lambda}$ of a particle, with $d$ its diameter, would be much less than one and implies that light absorption would exceed light scattering. The sensitivity of the radiative heat transfer to the shape of the soot aggregates - which are not considered - would therefore not be of first order.

\subsection{Numerical methods}

The particle number density function, governed by the Williams kinetic equation [34], is discretised in the Lagrangian dispersed phase solver by a finite number of tracked Dirac distributions or numerical particles, each having a numerical weight $w_{p}$. A numerical particle $p$ thereby represents $w_{p}$ physical particles with identical state vectors composed of the position $\mathbf{x}_{p}$, the velocity $\mathbf{V}_{p}$, the temperature $T_{p}$ and the radius $r_{p}$. For soot applications, the state vector of the numerical particles is updated at each iteration of duration $\Delta t$ through a collision and a transport step, detailed below.

\section{Collision step}

Collisions are described through a four-step algorithm, presented as part of the Supplementary materials, designed to reproduce the real physics of collisions from the tracking of numerical particles, the number of which being necessarily limited compared to the real number of physical particles. The underlying theory

\footnotetext{
${ }^{1}$ In terms of code architecture
} 
of the algorithm is out of the scope of this study and the interested reader is referred to the original work of Villedieu and Hylkema [35, 36, 37] for details.

According to the collision regime, the state vectors of particles 1 and 2 are modified as follows:

- For coalescence, the total volume of the particles is conserved. The numerical weight of particle 1 is therefore set to $w_{1}-w_{2}$ and the new state vector of particle 2 is computed through the system of equations (10):

$$
\left\{\begin{aligned}
\mathbf{x}_{2}^{c o a} & =a_{1} \mathbf{x}_{1}+a_{2} \mathbf{x}_{2}, \\
\mathbf{V}_{2}^{c o a} & =a_{1} \mathbf{V}_{1}+a_{2} \mathbf{V}_{2}, \\
T_{2}^{c o a} & =a_{1} T_{1}+a_{2} T_{2}, \\
r_{2}^{c o a} & =\left(\frac{3 \mathcal{V}^{c o a}}{4 \pi}\right)^{1 / 3},
\end{aligned}\right.
$$

where $a_{1}$ and $a_{2}$ are the ratios of the volumes $\mathcal{V}_{1}$ and $\mathcal{V}_{2}$ of particles 1 and 2 , respectively, to the one of the coalesced particle $\mathcal{V}^{c o a}=\mathcal{V}_{1}+\mathcal{V}_{2}$. It means that the result of the coalescence process is a spherical particle containing all the mass of the collision partners.

Note that, if both particles have originally the same numerical weight, their state vectors are taken equal to the one of the coalesced particle, and their numerical weights are halved.

- For "non-coalescence", the numerical weight of particle 1 is set to $w_{2}$, and its position and velocity are taken equal to the one of particle 2. It means that the result of the non-coalescence process is a set of spherical particles located at the same point with the same velocity, as if they were stuck to each other by a single contact point by analogy to aggregation.

Note that, if both particles have initially different numerical weights, a new particle, with a numerical weight equal to $w_{1}-w_{2}$ and a state vector identical to the one of particle 1 , has to be created first.

\section{Transport step}

The transport step describes the evolution, due to interactions with the gas phase, of the particles velocity, position and temperature on the one side, which is straightforward for tracers, and of the particles radius through chemical processes on the other side. At each time step, the new state vector - with superscript $n+1$ - of a numerical particle $p$ located in cell $c$ is calculated as a function of the former one - with superscript $n$ - through an explicit scheme as follows:

$$
\left\{\begin{array}{l}
\mathbf{V}_{p}^{n+1}=\mathbf{V}_{c}^{n}, \\
\mathbf{x}_{p}^{n+1}=\mathbf{x}_{p}^{n}+\mathbf{V}_{c}^{n} \Delta t, \\
T_{p}^{n+1}=T_{c}^{n} \\
m_{p}^{n+1}=m_{p}^{n}+\dot{\omega}_{p}^{n} \mathcal{V}_{c} \Delta t
\end{array}\right.
$$

where $\mathbf{V}_{c}$ and $T_{c}$ are the gas velocity and temperature in cell $c$, and $m_{p}$ the mass of the particle. The mass equation replaces here the radius equation for the sake of clarity, the new radius being inferred from equation 12 for spherical particles. The mass source term $\dot{\omega}$ will be discussed in Subsection 4.3.

$$
r_{p}^{n+1}=\sqrt[3]{\frac{3 m_{p}^{n+1}}{4 \pi \rho_{s}}}
$$




\section{Optimisation}

To reduce the computational cost of the Lagrangian solver, a quadtree-like procedure [38] grouping particles by radius $r$ and residence time $t_{r}$ - defined as the time required by a particle to leave the cell where it is located - is applied every $N_{t s}$ time steps. Its goal is to identify and suppress the numerical particles carrying redundant informations in each cell $c$ where the number of numerical particles $N_{n p, c}$ is higher than a constant $\widehat{N}_{n p}$. A dispersion criterion is used to that end in the two-dimensional $\left(r, t_{r}\right)$ - space, of primary interest here since the evolution of a particle in a cell is driven - among other parameters (cf. 4.3) - by its size and its interaction time with both the gas phase and the other particles.

In the reduction procedure described as part of the Supplementary materials, the sum of the particles volumes and the sum of the particles surfaces are conserved at the expense of their number, the reactive surface being of particular interest for the coupling with the gas phase. It is however worth noting that the conservation of all quantities is achieved when the maximum number of particles per cell $\widehat{N}_{n p}$ is sufficiently high (namely when $\bar{\sigma}_{q}$ tends towards zero in the Supplementary materials). The impact of $\widehat{N}_{n p}$ on the particle size distribution will therefore be discussed in Subsection 5.3.1. The particles in a cell are furthermore assumed to have close enough temperatures - even though particles transported from a neighbouring cell might introduce some discrepancies - so that their specific heat capacity is considered identical over the range of particle temperatures. The latter hypothesis allows to compute the temperature of each mean particle without the use of an iterative variable change.

\subsection{Momentum and energy feedback source terms}

For each time step $\Delta t$ and grid cell $c, \mathbf{S}_{q, c}^{d}$ and $S_{e_{t}, c}^{d}$ are calculated as:

$$
\begin{aligned}
& \mathbf{S}_{q, c}^{d}=-\frac{1}{\mathcal{V}_{c} \Delta t} \sum_{p \in \mathcal{P}_{c}(t)} w_{p} \int_{t}^{t+\Delta t} \underbrace{\left[\frac{d\left(m_{p} \mathbf{V}_{p}\right)}{d t}\right]}_{\text {Variation of the particle momentum }} d t \\
& S_{e_{t}, c}^{d}=-\frac{1}{\mathcal{V}_{c} \Delta t} \sum_{p \in \mathcal{P}_{c}(t)} w_{p} \int_{t}^{t+\Delta t}[\underbrace{\frac{1}{2} \frac{d\left(m_{p} \mathbf{V}_{p}^{2}\right)}{d t}}_{\begin{array}{c}
\text { Variation of the } \\
\text { particle kinetic energy }
\end{array}}+\underbrace{m_{p} c_{p} \frac{d T_{p}}{d t}}_{\uparrow}] d t, \\
& \text { Variation of the } \\
& \text { particle thermal energy }
\end{aligned}
$$

where $\mathcal{P}_{c}(t)$ is the set of particles located in cell $c$ at time $t$ and $c_{p}$ the specific heat capacity of particle $p$. Note that, even if the velocity and temperature of a particle are set to the ones of the gas phase at the transport step, their variations are to be known for the computation of the feedback source terms. Hence, both variables are modified during the collision and population reduction steps.

\section{Modelling of soot nucleation and evolution}

\subsection{Gas-phase chemistry}

Gas-phase chemistry is described in the CHARME solver using a reduced mechanism for ethylene combustion, successfully applied in previous studies [25, 39], which includes 43 species and 304 reactions. 


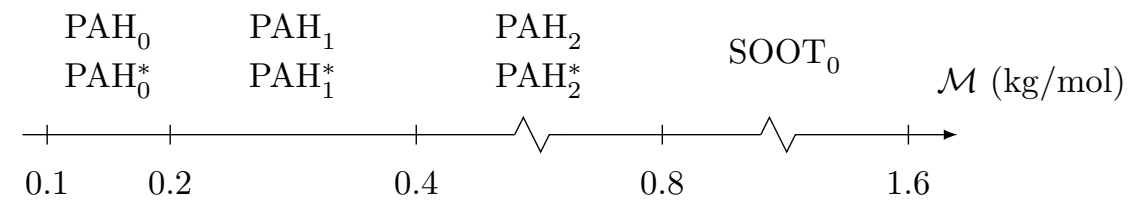

Figure 1: Definition of $\mathrm{PAH}_{\mathrm{i}}$ and $\mathrm{PAH}_{\mathrm{i}}^{*}$ sections [39].

\subsection{PAH modelling}

Polycyclic aromatic hydrocarbons are modelled in the CHARME solver using the sectional method proposed by Di Domenico et al. [24], Blacha et al. [25], and recently updated by Eberle et al. [39]. Three sections of $\mathrm{PAH}$ and $\mathrm{PAH}$ radicals (respectively noted $\mathrm{PAH}_{\mathrm{i}}$ and $\mathrm{PAH}_{\mathrm{i}}^{*}, 0 \leq i \leq 2$ ), whose molecular weights range from $0.1 \mathrm{~kg} / \mathrm{mol}$ to $0.8 \mathrm{~kg} / \mathrm{mol}$, are considered (see Fig. 1). A soot section $\mathrm{SOOT}_{0}$ is added for both the derivation of the reaction mechanism and the modelling of the nucleated soot particles.

The atomic composition, the thermodynamic properties, as well as the viscosity for each section are calculated from reference polycyclic aromatic hydrocarbons [25, 39]. For the sake of simplicity, the binary diffusion coefficients of $\mathrm{PAH}_{\mathrm{i}}$ and $\mathrm{PAH}_{\mathrm{i}}^{*}$ are here assumed to be equal to the ones of the smallest $\mathrm{PAH}$ (benzene) and of its radical (phenyl), respectively. Their diffusion coefficients are computed through equation (5).

\subsection{1. $\mathrm{PAH}_{\mathrm{i}}-\mathrm{PAH}_{\mathrm{i}}^{*}$ reaction mechanism}

The reaction mechanism is composed of five sub-mechanisms [25, 39]: PAH-gas phase interaction, PAH collisions, $\mathrm{PAH}$ growth by addition of $\mathrm{C}_{2} \mathrm{H}_{2}$, PAH dehydrogenation and $\mathrm{PAH}$ oxidation by $\mathrm{O}, \mathrm{OH}$ and $\mathrm{O}_{2}$. $\mathrm{PAH}_{\mathrm{i}}$ and $\mathrm{PAH}_{\mathrm{i}}^{*}$ being minor species, their third-body efficiencies are additionally set to zero in the gas phase reaction mechanism for the present study.

\subsubsection{Soot nucleation rate}

Soot nucleation is modelled through the collision of $\mathrm{PAH}_{2}$ and $\mathrm{PAH}_{2}^{*}$ with the other sections [39]:

$$
\begin{aligned}
& \mathrm{PAH}_{\mathrm{i} \leq 2}^{*}+\mathrm{PAH}_{2}^{*} \rightarrow \nu_{1} \mathrm{PAH}_{2}+\nu_{2} \mathrm{SOOT}_{0}+\nu_{3} \mathrm{H}_{2}, \\
& \mathrm{PAH}_{\mathrm{i} \leq 2}^{*}+\mathrm{PAH}_{2} \rightarrow \nu_{1} \mathrm{PAH}_{2}+\nu_{2} \mathrm{SOOT}_{0}+\nu_{3} \mathrm{H}_{2}+\mathrm{H}, \\
& \mathrm{PAH}_{\mathrm{i} \leq 1}+\mathrm{PAH}_{2}^{*} \rightarrow \nu_{1} \mathrm{PAH}_{2}+\nu_{2} \mathrm{SOOT}_{0}+\nu_{3} \mathrm{H}_{2}+\mathrm{H},
\end{aligned}
$$

where the stoichiometric coefficients $\nu_{1}, \nu_{2}$ and $\nu_{3}$, calculated using the work of Pope et al. [40], ensure the conservation of both mass and elements. The reaction rates are computed similarly to the study by Blacha et al. [25] and the reaction efficiencies from the work of Eberle et al. [39] are used without modification.

The mass production rate of $\mathrm{SOOT}_{0}$ - the soot nucleation rate $\dot{\omega}_{0}$ - is computed as for a standard gas species [31] assuming a molecular weight $\mathcal{M}_{0}$ equal to $1.2 \mathrm{~kg} / \mathrm{mol}$ and transmitted to the dispersed phase solver SPARTACCUS at each time step, so that no transport equation is required for the latter species. Also note that the soot volume fraction, lower than $1 \times 10^{-6}$ in the simulation cases hereinafter (see Fig. 5), is far below usual validity limits of two-phase dispersed flow hypotheses [41] and therefore neglected in the CHARME solver.

\subsection{Soot particles modelling}

Soot particles are modelled in the SPARTACCUS solver by solid spheres, as mentioned in Section 3. Their density $\rho_{s}$ is assumed constant - typically between 1700 and $2000 \mathrm{~kg} / \mathrm{m}^{3}$ in the litterature - and 
chosen equal to $1800 \mathrm{~kg} / \mathrm{m}^{3}$ [22] hereinafter. From the preliminary work of Blacha et al. [25], their specific heat capacity is taken equal to the one of acetylene.

\subsubsection{Particles nucleation}

For each time step $\Delta t$ and grid cell $c$, the mass of soot $\bar{m}_{c}$ and the number of physical particles $N_{p p, c}$ to be injected are calculated as follows:

$$
\begin{aligned}
& \bar{m}_{c}=\dot{\omega}_{0, c} \mathcal{V}_{c} \Delta t, \\
& N_{p p, c}=\frac{m_{c} \mathcal{N}_{a}}{\mathcal{M}_{0}},
\end{aligned}
$$

where $\mathcal{N}_{a}$ is the Avogadro number.

If $\bar{m}_{c}$ has a non-zero value, a single numerical particle is generally injected with a numerical weight equal to $N_{p p, c}$. Its radius $r_{0}$ is given as a function of $\rho_{s}$ and $\mathcal{M}_{0}$ by (18):

$$
r_{0}=\left(\frac{3 \mathcal{M}_{0}}{4 \pi \mathcal{N}_{a} \rho_{s}}\right)^{1 / 3}=0.64 \mathrm{~nm}
$$

Its velocity and temperature are initialised to the values in cell $c$, and its position is determined randomly from the equivalent cell diameter. In the present study, the numerical weight $w_{p}$ of each particle $p$ is nevertheless bounded at injection to ensure the representativeness of the particles population and limit the number of non-significant subjects. Considering an initial mass $\bar{m}_{c}$, a numerical particle of maximum numerical weight is therefore injected until $\bar{m}_{c}$ reaches zero or $w_{p}$ stands below the lower bound. Eventually, the remaining non-injectable mass is stored for a use in the next time step.

$r_{0}$ is additionally used as a removal criterion for the non-significant particles at the end of the transport step. Hence, particles are suppressed if their radius becomes lower than $2^{-1 / 3} r_{0}$, namely if their volume is at least twice as small as the one of the nucleated soot particles.

\subsubsection{Chemically-driven evolution}

\section{General formulation}

The generic reaction of a soot particle SOOT with a species R is first considered:

$$
\mathrm{SOOT}+\nu_{\mathrm{R}} \mathrm{R} \rightarrow \mathrm{SOOT}^{\#}+\nu \mathrm{H}_{2}+\nu_{\mathrm{P}} \mathrm{P}
$$

where $\mathrm{SOOT}^{\#}$ is the state of SOOT after reaction with $\mathrm{R}$, and $\mathrm{P}$ the associated product. The term $\nu \mathrm{H}_{2}$ ensures mass conservation.

The molecular weight of SOOT\# and the stoichiometric coefficient $\nu$ are determined iteratively at each time step as follows:

$$
\mathcal{M}^{\#}=n_{C}^{\#}\left[\mathcal{M}_{C}+H / C\left(\mathcal{M}^{\#}\right) \mathcal{M}_{H}\right]
$$

and

$$
\nu=\frac{\mathcal{M}-\mathcal{M}^{\#}+\nu_{R} \mathcal{M}_{R}-\nu_{P} \mathcal{M}_{P}}{2 \mathcal{M}_{H}}
$$

where $n_{C}^{\#}$ is the number of carbon atoms in SOOT\#, calculated from the one in SOOT, R and $\mathrm{P}$, and $\mathcal{M}$ the molecular weight of SOOT. $H / C$ is the ratio of hydrogen to carbon atoms in a soot particle, computed 
in the present study through the correlation introduced by Blacha et al. [25] from the work of Richter et al. [23]:

$$
H / C=0.4405 \mathcal{M}^{-0.10524},
$$

with $\mathcal{M}$ in $\mathrm{kg} / \mathrm{mol}$.

For a particle located in cell $c$ at time $t$, the progress rate $\mathcal{Q}$ of reaction (19) is given by equation (23),

$$
\mathcal{Q}=\mathcal{K}[\mathrm{R}]_{c}^{\nu_{R}} \underbrace{\frac{1}{\mathcal{N}_{a} \mathcal{V}_{c}},}_{\text {Apparent concentration of SOOT }}
$$

with $\mathcal{K}$ the reaction rate constant and $[\mathrm{R}]_{c}$ the concentration of species $\mathrm{R}$ in $c$.

The evaluation of the reaction rate constants for each evolution model is detailed hereinafter, with $[\mathcal{Y}]_{c}$ the set of gas species concentrations in $c$. The oxidation models are presented for the sake of completeness, however it is worth noting that their contribution to soot particles evolution in the following test cases is not significant.

\section{Surface growth by addition of $\mathrm{C}_{2} \mathrm{H}_{2}$}

For surface growth by addition of $\mathrm{C}_{2} \mathrm{H}_{2}$, generic reaction (19) writes:

$$
\mathrm{SOOT}+\mathrm{C}_{2} \mathrm{H}_{2} \rightarrow \mathrm{SOOT}^{\#}+\nu \mathrm{H}_{2} .
$$

The reaction rate constant is calculated using the model of Frenklach and Wang [42, 43] as:

$$
\mathcal{K}_{\mathrm{C}_{2} \mathrm{H}_{2}}=\mathcal{K}_{\mathrm{C}_{2} \mathrm{H}_{2}, s}\left(T_{c}\right) \alpha \chi_{s}\left(T_{c},[\mathcal{Y}]_{c}\right) S,
$$

where $\mathcal{K}_{\mathrm{C}_{2} \mathrm{H}_{2}, s}$ is a per-site reaction rate constant, $\alpha$ the fraction of reactive surface sites, $\chi_{s}$ the number density of surface sites and $S$ the particle surface.

\section{Oxidation by $\mathrm{O}_{2}$}

For oxidation by $\mathrm{O}_{2}$, generic reaction (19) reads:

$$
\mathrm{SOOT}+\mathrm{O}_{2} \rightarrow \mathrm{SOOT}^{\#}+\nu \mathrm{H}_{2}+2 \mathrm{CO} .
$$

The reaction rate constant, also computed using the model of Frenklach and Wang, is given by equation (27).

$$
\mathcal{K}_{\mathrm{O}_{2}}=\mathcal{K}_{\mathrm{O}_{2}, s}\left(T_{c}\right) \alpha \chi_{s}\left(T_{c},[\mathcal{Y}]_{c}\right) S
$$

\section{Oxidation by $\mathrm{OH}$}

For oxidation by $\mathrm{OH}$, generic reaction (19) writes:

$$
\mathrm{SOOT}+\mathrm{OH} \rightarrow \mathrm{SOOT}^{\#}+\nu \mathrm{H}_{2}+\mathrm{CO} .
$$

The reaction rate constant is calculated using the model of Neoh et al.[44] as:

$$
\mathcal{K}_{\mathrm{OH}}=\mathcal{N}_{a} \gamma_{\mathrm{OH}}\left(\frac{\mathcal{R} T_{c}}{2 \pi \mathcal{M}_{\mathrm{OH}}}\right)^{0.5} S,
$$

where the collision efficiency $\gamma_{\mathrm{OH}}$ is equal to 0.13 . 


\section{Condensation of $\mathrm{PAH}_{\mathrm{i}}$ and $\mathrm{PAH}_{\mathrm{i}}^{*}$}

By analogy to the nucleation reactions (15), the reaction product $\mathrm{P}$ of a soot particle with a $\mathrm{PAH}$ radical in (19) is chosen to be $\mathrm{H}$. Therefore, the generic reaction reads for the condensation of $\mathrm{PAH}_{\mathrm{i}}^{*}$ :

$$
\mathrm{SOOT}+\mathrm{PAH}_{\mathrm{i} \leq 2}^{*} \rightarrow \mathrm{SOOT}^{\#}+\nu^{*} \mathrm{H}_{2}+\mathrm{H},
$$

and can be written for the condensation of $\mathrm{PAH}_{\mathrm{i}}$ as:

$$
\mathrm{SOOT}+\mathrm{PAH}_{\mathrm{i} \leq 2} \rightarrow \mathrm{SOOT}^{\#}+\nu \mathrm{H}_{2} .
$$

The reaction rate constant is computed for both as follows:

$$
\mathcal{K}_{\mathrm{PAH}^{(*)}}=k_{v d w} \mathcal{N}_{a} \gamma_{\mathrm{PAH}^{(*)}} \beta_{i, s}^{*}\left(d_{c, i}, S, T_{c}, l_{c}, \mu_{c}\right),
$$

where $k_{v d w}$ is the van der Waals enhancement factor, corresponding to the increase of the collision rate due to the attractive van der Waals forces between the collision partners, equal to 2.2 [45]. $\beta_{i, s}^{*}$ is the collision frequency and $\gamma_{\mathrm{PAH}^{(*)}}$ the collision efficiency, initially fixed to 0.3 [39] and discussed in Subsection 5.4.

The collision frequency between $\mathrm{PAH}_{\mathrm{i}}$ or $\mathrm{PAH}_{\mathrm{i}}^{*}$ and a soot particle is determined using the general Fuchs formulation [46], including transition from the free molecular regime to the continuum regime with increasing particle diameter. Apart from $S, \beta_{i, s}^{*}$ is a function of the $\mathrm{PAH}$ or $\mathrm{PAH}^{*}$ collision diameter $d_{c, i}$, as well as of the gas temperature $T_{c}$, mean free path $l_{c}$ and viscosity $\mu_{c} . d_{c, i}$, in Angström, is calculated for both $\mathrm{PAH}_{\mathrm{i}}$ and $\mathrm{PAH}_{\mathrm{i}}^{*}$ through the correlation of Frenklach and Wang [12]:

$$
d_{c, i}=1.395 \sqrt{2} \sqrt{n_{C i}}[\AA],
$$

where $n_{C i}$ is the number of carbon atoms of the species $\mathrm{PAH}_{\mathrm{i}}^{(*)}$.

\section{Particle mass source term}

The mass source term of a particle is calculated as a function of the mass reaction rates associated to each evolution model $M$ as:

$$
\dot{\omega}=\sum_{M}\left(\mathcal{M}_{M}^{\#}-\mathcal{M}\right) \mathcal{Q}_{M} .
$$

The mass of a particle being positive, the term $\dot{\omega} \mathcal{V}_{c} \Delta t$ in equation 11 has to be higher than $-m$, with $m$ the original mass of the particle. The reaction rate of all evolution models is therefore corrected by a factor $\epsilon$ defined by equation (35),

$$
\epsilon= \begin{cases}m /\left|\dot{\omega} \mathcal{V}_{c} \Delta t\right| & \text { if } \dot{\omega} \mathcal{V}_{c} \Delta t<-m, \\ 1 & \text { otherwise },\end{cases}
$$

so that the apparent reaction time associated to the particle for the feedback to the gas phase becomes $\epsilon \Delta t$.

\section{Feedback source terms}

For a given evolution model $M$ and a given particle $p$, the mass source term of species $\mathrm{R}, \mathrm{H}_{2}$ and $\mathrm{P}$ is expressed as follows:

$$
\left(\begin{array}{c}
\dot{\omega}_{\mathrm{R}} \\
\dot{\omega}_{\mathrm{H}_{2}} \\
\dot{\omega}_{\mathrm{P}}
\end{array}\right)_{p, M}=w_{p} \epsilon_{p} \mathcal{Q}_{p, M}\left(\begin{array}{c}
-\mathcal{M}_{\mathrm{R}} \nu_{\mathrm{R}} \\
\mathcal{M}_{\mathrm{H}_{2}} \nu_{p} \\
\mathcal{M}_{\mathrm{P}} \nu_{\mathrm{P}}
\end{array}\right)_{M},
$$


and the feedback source term $S_{k, c}^{d}$ of the dispersed phase on the gas phase in cell $c$ as:

$$
S_{k, c}^{d}=\sum_{M} \sum_{p \in \mathcal{P}_{c}(t)} \dot{\omega}_{k, p, M}, \quad k=\mathrm{R}, \mathrm{H}_{2}, \mathrm{P} .
$$

$S_{k, c}^{d}$, along with the aforementioned source terms, allows a complete two-way coupling of the gas-phase and dispersed phase solvers.

\subsubsection{Collision parameters}

While soot particles are assumed to behave as tracers at the macroscopic scale, their trajectory at the mesoscopic one is driven by Brownian motion, namely by their interaction with the surrounding molecules of the gas phase. Hence, the collision frequency $\beta_{12}$ (cf. Supplementary materials) of two soot particles is calculated as:

$$
\beta_{12}=k_{v d w} \beta_{12}^{*},
$$

where $\beta_{12}^{*}$ is the collision frequency function of Brownian motion introduced in the work of Fuchs [46].

$d_{\text {crit }}$ is also fixed to $13 \mathrm{~nm}$ as a first guess, one order of magnitude higher than the diameter of nucleated particles.

\section{Validation and discussion}

\subsection{Experimental test cases}

The ethylene-air flames simulated in the present study are burner-stabilised laminar premixed flames at atmospheric pressure investigated by Xu et al. [32] on a McKenna burner. Experimental measurements are advantageously available at three fuel equivalence ratios $\phi$, especially delivered as profiles of the major gas species mole fraction, the primary particle diameter, and the soot volume fraction. The operating conditions for the three test cases are reported in Tab. 1.

Table 1: Test cases inlet conditions.

\begin{tabular}{cc|c|c|c}
\hline Equivalence ratio & {$[-]$} & 2.34 & 2.64 & 2.94 \\
\hline Cold gas velocity & {$[\mathrm{cm} / \mathrm{s}]$} & 6.73 & 6.73 & 6.73 \\
Cold gas temperature & {$[\mathrm{K}]$} & 298 & 298 & 298 \\
$X_{\mathrm{C}_{2} \mathrm{H}_{4}}$ & {$[\%]$} & 14.08 & 15.60 & 17.0 \\
$X_{\mathrm{O}_{2}}$ & {$[\%]$} & 18.05 & 17.73 & 17.4 \\
$X_{\mathrm{N}_{2}}$ & {$[\%]$} & 67.84 & 66.67 & 65.6 \\
\hline
\end{tabular}

Soot volume fraction profiles measured by intrusive sampling are given in Fig. 5 for indicative purposes only. Also note that the diameter of the transported spherical particles will be compared to the measured primary particle diameter in the following, which is consistent with the collision model used in the present study.

\subsection{Numerical setup}

The computational domain is 2D, $40 \mathrm{~mm}$ long and $1.5 \mathrm{~mm}$ wide. The mesh grid contains a total of 210 cells and is refined within the flame front, with a single cell in the transverse direction. The inlet boundary conditions are deduced from Tab. 1 for each test case. Atmospheric pressure combined with non-reflective 
acoustic conditions are imposed at the outlet, symmetry conditions are used elsewhere. Temperature profiles suggested by the International Sooting Flame Workshop [47] are additionally prescribed at each iteration of the CHARME solver. The time step is fixed to $1 \mu \mathrm{s}$ for both the gas phase and the dispersed phase solver, and the simulated physical time is chosen large enough to reach a steady-state solution.

A preliminary computation with Cantera [48] was carried out at each equivalence ratio. The solution obtained by only considering the gas phase reaction mechanism allows here to define a proper initial state for the CEDRE calculation.

\subsection{Evaluation of the optimisation algorithm}

\subsubsection{Reduction intensity of the optimisation algorithm}

In order to evaluate the statistical convergence of the quadtree-like algorithm, six cases are simulated at the intermediate equivalence ratio $\phi=2.64$. All are identical, except for the maximum number $\widehat{N}_{n p}$ of numerical particles per cell, successively limited to 64, 128, 256, 512, 1024 and 2048 every 100 time steps. In each case, the properties of the particles are measured $20 \mathrm{~mm}$ and $30 \mathrm{~mm}$ above the burner for comparison purposes, these two locations being denoted as measurement points in the following.

\section{Mean and standard deviation}

Figure 2 compares the mean particle diameter $d_{10}$ and its standard deviation $\sigma_{d}$ at both measurement points and for all cases. For the sake of simplicity, the variables are normalised by the values reached at $\widehat{N}_{n p}=2048$, this simulation being considered as a reference given the very good convergence of $d_{10}$ and $\sigma_{d}$ with increasing $\widehat{N}_{n p}$.

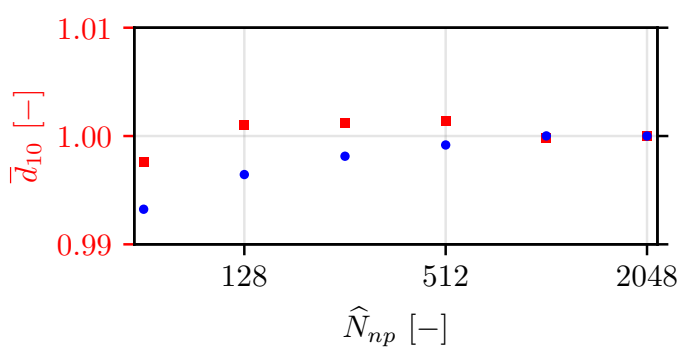

(a) $H A B=20 \mathrm{~mm}$

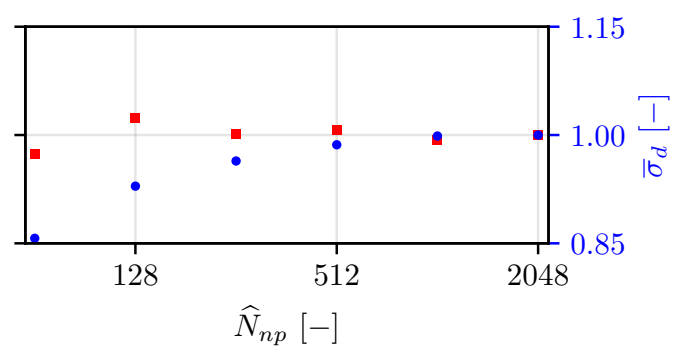

(b) $H A B=30 \mathrm{~mm}$

Figure 2: Normalised mean particle diameter $(\square)$ and standard deviation $(\mathbf{)})$ as a function of $\widehat{N}_{n p}$ at the measurement points located $20 \mathrm{~mm}$ and $30 \mathrm{~mm}$ above the burner. For $\widehat{N}_{n p}=2048, d_{10}=25.4 \mathrm{~nm}$ and $\sigma_{d}=2.64 \mathrm{~nm}$ (a), $d_{10}=26.9 \mathrm{~nm}$ and $\sigma_{d}=2.60 \mathrm{~nm}(\mathrm{~b})$.

The gap between $d_{10}$ and its reference value remains lower than $1 \%$. In contrast, the gap reaches $14 \%$ for $\sigma_{d}$ at the point located $30 \mathrm{~mm}$ above the burner for the lowest value of $\widehat{N}_{n p}$, i.e. 64 . Thus, while the algorithm allows to preserve the mean of the particle size distribution, even for low values of the parameter $\widehat{N}_{n p}$, the gap for the standard deviation increases with the decrease of $\widehat{N}_{n p}$. This behaviour, intrinsically related to the reduction of the particles population, is not surprising. $\widehat{N}_{n p}$ should just be set as a compromise between the computational cost and the accuracy in predicting the standard deviation, depending on the application. In the following, it will be taken equal to 256, which is assumed sufficient for the comparison with the experimental measurements, only available upstream of the second point. 


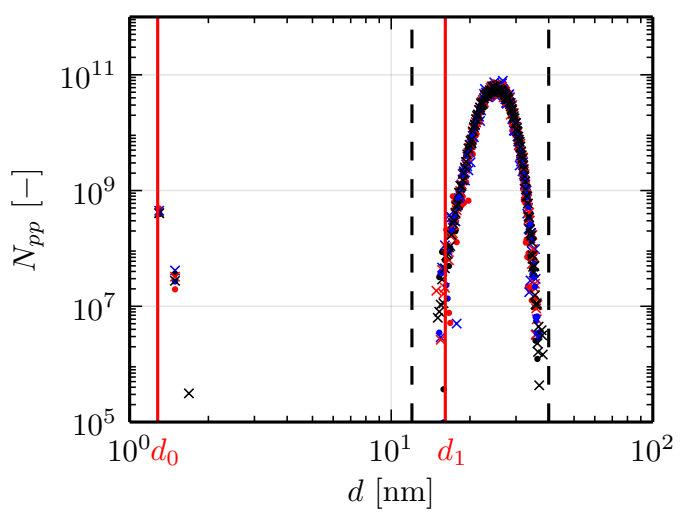

(a) $H A B=20 \mathrm{~mm}$

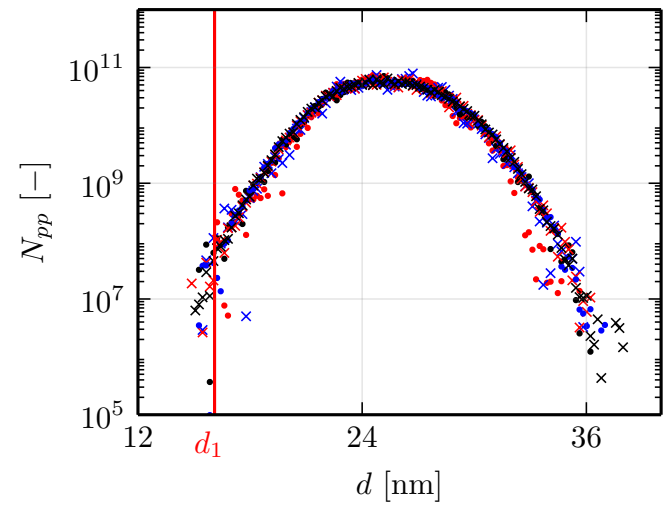

(c) $H A B=20 \mathrm{~mm}$

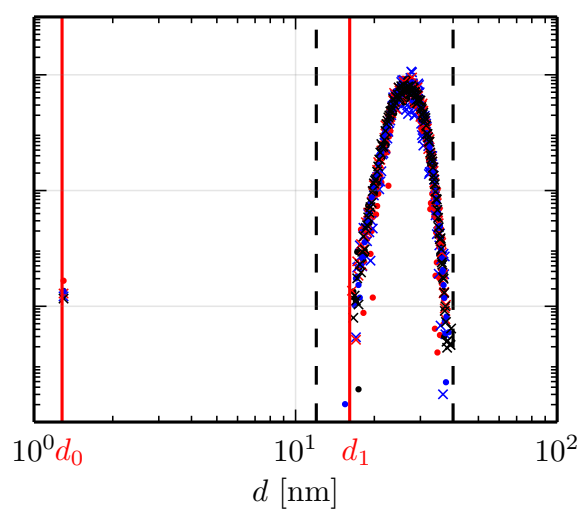

(b) $H A B=30 \mathrm{~mm}$

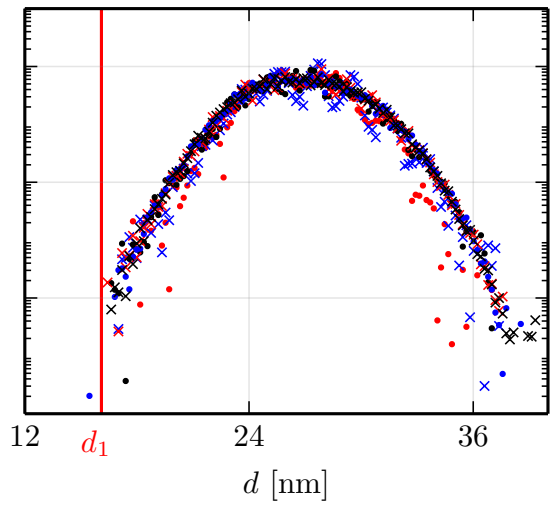

(d) $H A B=30 \mathrm{~mm}$

Figure 3: Particle size distribution as a function of $\widehat{N}_{n p}$ at the measurement points located $20 \mathrm{~mm}$ and $30 \mathrm{~mm}$ above the burner $(\boldsymbol{O}=64, X=128, \boldsymbol{O}=256, X=512, \boldsymbol{O}=1024, X=2048)$. The bottom figures are a zoom of the top ones in the region delimited by the black dashed lines. 


\section{Particle size distribution}

Figure 3 shows the particle size distribution at both measurement points and for all cases. As expected, the deviation from the reference case varies inversely with the parameter $\widehat{N}_{n p}$. Nonetheless, even for strong limitations of the maximum number of particles per cell, the shape of the distribution - representative of the individual history of the particles - is preserved. Two peaks are visible at each point. The first one matches the diameter of nucleated soot particles $d_{0}$. The second one is lower bounded by the diameter $d_{1}=2^{1 / 3} d_{\text {crit }}$, which corresponds to the diameter of a particle formed through the coalescence of two particles with a diameter equal to $d_{c r i t}$. It indicates that coalescence stops very early downstream of the flame. The evolution of the soot particles, evidenced by the weakening of the first peak and the shift of the second peak towards higher diameters with increasing height above the burner (HAB), is associated to surface growth and condensation, as well as to coalescence of the remaining or newly nucleated small particles with the bigger ones.

\subsubsection{Reduction frequency of the optimisation algorithm}

The quadtree-like algorithm is now successively applied every $N_{t s}$ time steps, with $N_{t s}=1,10$ and 1000 , $\widehat{N}_{n p}$ being fixed to 256 . The three cases are simulated at the intermediate equivalence ratio $\phi=2.64$ to assess the impact of this reduction frequency on the first and second order statistical moments of the particle size distribution.

Figure 4 compares the mean particle diameter $d_{10}$ and its standard deviation $\sigma_{d}$ at both measurement points and for all cases. The variables are normalised by the values previously obtained for $N_{t s}=100$ for the sake of clarity.

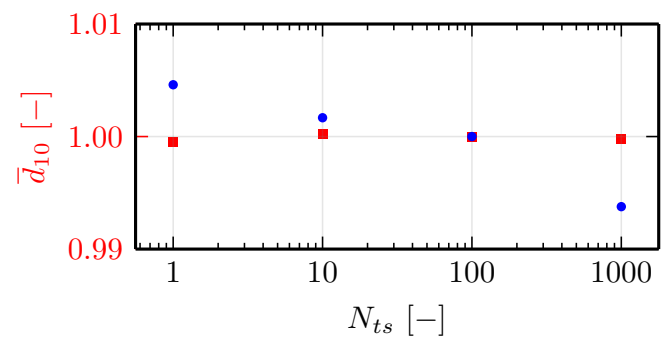

(a) $H A B=20 \mathrm{~mm}$

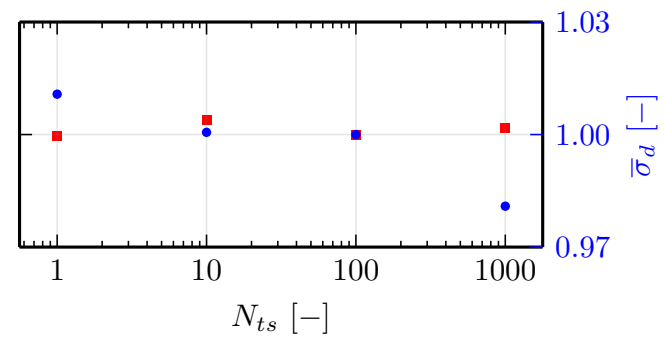

(b) $H A B=30 \mathrm{~mm}$

Figure 4: Normalised mean particle diameter $(\square)$ and standard deviation $(\mathbf{O})$ as a function of $N_{t s}$ at the measurement points located $20 \mathrm{~mm}$ and $30 \mathrm{~mm}$ above the burner. For $N_{t s}=100, d_{10}=25.4 \mathrm{~nm}$ and $\sigma_{d}=2.57 \mathrm{~nm}(\mathrm{a}), d_{10}=26.9 \mathrm{~nm}$ and $\sigma_{d}=2.50 \mathrm{~nm}(\mathrm{~b})$.

The gap between $d_{10}$ or $\sigma_{d}$ and their reference values always remains smaller than $2 \%$, so that the influence of the reduction frequency is considered to be negligible within the explored range of values. The parameter $N_{t s}$ will therefore be taken equal to 100 in Subsection 5.4 to keep the computational cost moderate as discussed below.

\subsubsection{Remark on the computational cost}

As a guide for comparison to sectional methods or methods of moments, the computational cost of the Lagrangian solver represents $38 \%$ of the total cost for $\widehat{N}_{n p}=256$ and $N_{t s}=100,53$ transport equations being solved for the gas-phase. For $N_{t s}=100$, the mean computational cost of the Lagrangian solver 
decreases from $4.5 \mathrm{~s} /$ time step for $\widehat{N}_{n p}=2048$ to $0.64 \mathrm{~s} /$ time step for $\widehat{N}_{n p}=64$, due to the reduced size of the particles population. For $\widehat{N}_{n p}=256$, the mean computational cost of the Lagrangian solver increases from $0.89 \mathrm{~s} /$ time step for $N_{t s}=100$ to $2.3 \mathrm{~s} /$ time step for $N_{t s}=1000$, due to the growth of the particles population between two reductions. From $N_{t s}=100$ to $N_{t s}=1$, the mean computational cost also increases following the high number of reduction events, for which monitoring files are additionally written.

Note that the performance of the Lagrangian solver could be improved by dynamic load balancing in the case of massively parallel simulations.

\subsection{Comparison with experimental measurements}

\subsubsection{Soot volume fraction and particle diameters}

Figures 5 and 6 present the profiles of soot volume fraction $f_{v}$ and mean particle diameter $d_{10}$ at the three fuel equivalence ratios. From a global point of view, the model proves its ability to reproduce the growth of both quantities with increasing values of $\phi$. Fairly good agreement with the measurements is especially noticed for the soot volume fraction at $\phi=2.64$ and $\phi=2.94$. However, while the mean particle diameter is correctly predicted in the first case, it is underestimated in the second one. The sensitivity of the model to the limitation of coalescence processes will therefore be discussed below. At $\phi=2.34$, the soot volume fraction and the mean particle diameter are both underrated. The temperature profile being fixed, the influence of two parameters has here to be investigated, namely the collision efficiency $\gamma_{\mathrm{PAH}^{(*)}}$, introduced in Subsection 4.3, and the kinetic constants for $\mathrm{C}_{2} \mathrm{H}_{2}$ addition. Note that similar discrepancies are also observed by Eberle et al. [39] at low fuel equivalence ratios for a comparable configuration and an identical nucleation mechanism, showing that an upgrade of the nucleation model could be considered. Such reflections are however beyond the scope of the present study.

\section{Limitation of coalescence processes}

Figure 6 show the profile of $d_{10}$ at the three fuel equivalence ratios for two additional values of the critical diameter $d_{c r i t}$, i.e. $9.1 \mathrm{~nm}$ and $18 \mathrm{~nm}$. As expected, the increase of $d_{\text {crit }}$ promotes coalescence, which augments the size of the particles. At $\phi=2.34$, coalescence stops far downstream of the flame zone, so that the impact of the critical diameter is only discernible close to the outlet. Note that the profiles of $f_{v}$ are not presented since not deeply affected by the critical diameter.

\section{Collision efficiency $\gamma_{\boldsymbol{P A} \boldsymbol{H}^{(*)}}$}

Figure 7 compare the profiles of $f_{v}$ and $d_{10}$ at $\phi=2.34$ for two values of the collision efficiency, i.e. 0.3 (reference simulation) and 1.0. Both quantities increase with $\gamma_{\mathrm{PAH}^{(*)}}$ due to improved condensation. The consumption of the PAH molecules and radicals also modifies the distribution of these species above the burner, which reduces the soot nucleation rate, displayed in Fig. 7, and the particle number density. The growth of the particles however overcompensates this phenomenon, as evidenced by the elevation of the soot volume fraction. For the sake of completeness, Fig. 7 shows the aforementioned profiles for two additional combinations of collision efficiencies, i.e. 1.0 for PAH molecules with 0.3 for radical species, and vice versa. The introduction of such an asymmetry demonstrates that PAH condensation onto soot particles overrides that attributed to $\mathrm{PAH}^{*}$. 


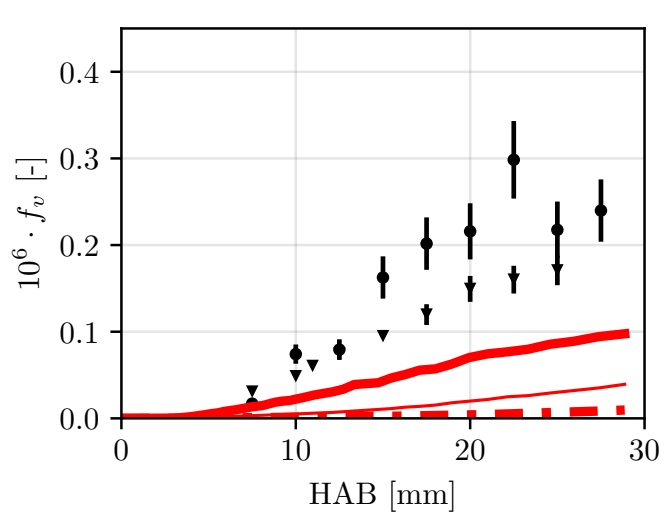

(a) $\phi=2.34$

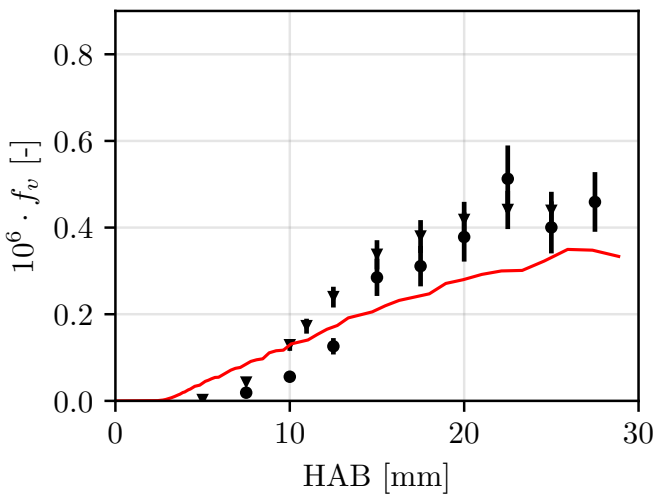

(b) $\phi=2.64$

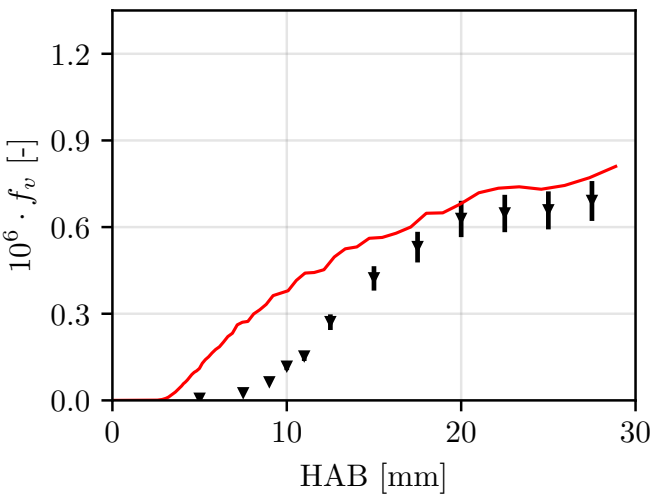

(c) $\phi=2.94$

Figure 5: Soot volume fraction along height above the burner: $\mathrm{Xu}$ - laser extinction $(\boldsymbol{\nabla}), \mathrm{Xu}$ - sampling $(\mathbf{O}), \mathrm{CE}-$ $\mathrm{DRE}$ at $d_{\text {crit }}=13 \mathrm{~nm}(-)$, CEDRE at $\widehat{T}-50 \mathrm{~K}(-)$ and $\widehat{T}+50 \mathrm{~K}(-\cdot-\boldsymbol{)})$.

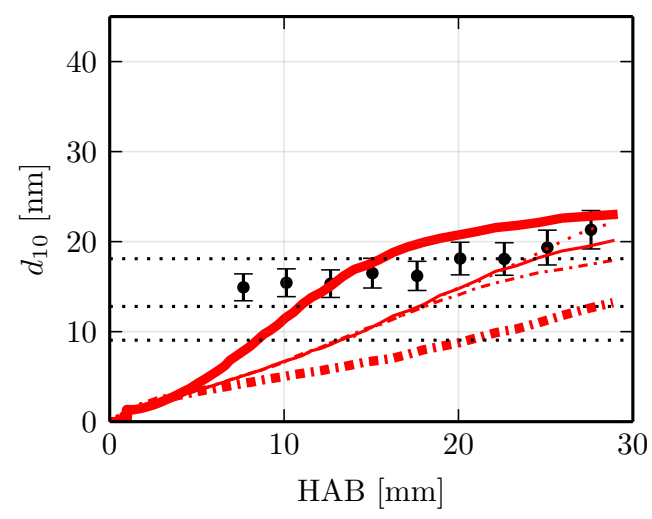

(a) $\phi=2.34$

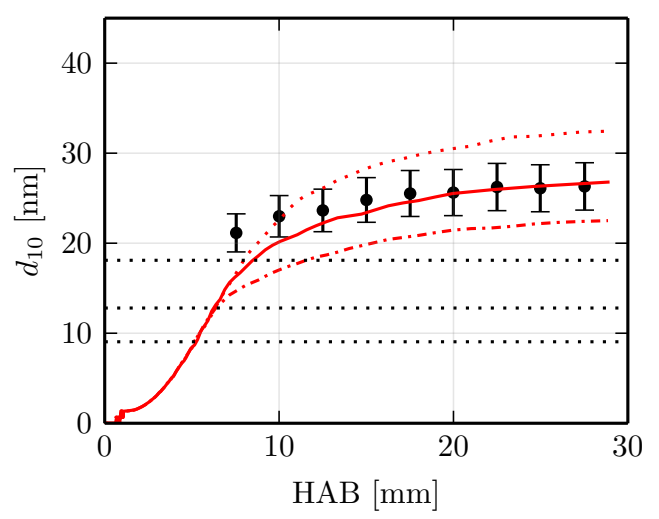

(b) $\phi=2.64$

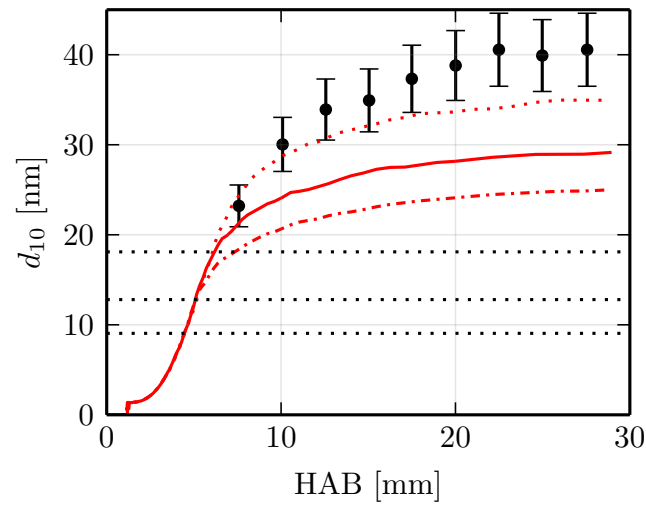

(c) $\phi=2.94$

Figure 6: Mean particle diameter along height above the burner: $\mathrm{Xu}(\mathbf{O})$, CEDRE at $d_{\text {crit }}=9.1 \mathrm{~nm}(-\cdot-)$, $13 \mathrm{~nm}(-), 18 \mathrm{~nm}(\cdots)$, CEDRE at $\widehat{T}-50 \mathrm{~K}(-)$ and $\widehat{T}+50 \mathrm{~K}(--)$. The critical diameters are reminded by the black dashed lines.

Table 2: Adiabatic flame temperatures (estimated from a preliminary equilibrium calculation carried out with Cantera and only considering the gas phase reaction mechanism).

\begin{tabular}{cc|c|c|c}
\hline Equivalence ratio & {$[-]$} & 2.34 & 2.64 & 2.94 \\
\hline $\begin{array}{c}\text { Estimated adiabatic } \\
\text { flame temperature }\end{array}[\mathrm{K}]$ & 1728 & 1583 & 1449 \\
\hline
\end{tabular}




\section{Kinetic of $\mathrm{C}_{2} \mathrm{H}_{2}$ addition}

As indicated in Subsection 4.3, the reaction rate constant for $\mathrm{C}_{2} \mathrm{H}_{2}$ addition is proportional to the fraction of active surface sites $\alpha$. In the present study, $\alpha$ is calculated through the correlation of Kazakov et al. [43] from the maximum flame temperature assimilated to the adiabatic one $T_{a d}$. This is reported in Tab. 2 for the three fuel equivalence ratios investigated. At $\phi=2.34$, no significant sensitivity of the volume fraction and mean particle diameter to variations of the temperature value used could however be highlighted. Hence, the present study focuses on their sensitivity to the pre-exponential factor $A_{s g}$ of the per-site reaction rate constant.

Figure 8 shows the profiles of soot volume fraction and mean particle diameter at $\phi=2.34$ for $A_{s g} / A_{s g}^{0}$ equal to 1 (reference simulation) and 10. As expected, both $f_{v}$ and $d_{10}$ increase with the pre-exponential factor. Nevertheless, the higher consumption of acetylene due to surface growth has no discernible impact on its distribution above the burner. The reduction of the soot nucleation rate, although limited, is therefore directly linked to the stronger consumption of PAH molecules and radicals with increasing particle size in the flame zone.

\section{Discussion}

According to the sensitivity analysis, a critical diameter equal to $18 \mathrm{~nm}$ offers the best compromise for the three fuel equivalence ratios, at least as a first approximation. As a result, this value is retained, although its validity for different fuels, combustion and turbulence regimes should be investigated in the future.

Concerning the collision efficiencies for $\mathrm{PAH} / \mathrm{PAH}^{*}$ condensation and the kinetic constants for $\mathrm{C}_{2} \mathrm{H}_{2}$ addition, no change in the standard values can be justified without further validation. The reported results however highlight the major role of PAH condensation on both particle nucleation and growth.

\subsubsection{Temperature profiles}

In order to emphasize the sensitivity of the soot formation and evolution processes to the temperature level, the profile imposed at $\phi=2.34$ is rescaled through an homothetic transformation, so that profiles with maximum values varying of $\pm 50 \mathrm{~K}$ compared to the initial one $(\widehat{T})$ can be tested. Strictly speaking, for a burner-stabilised flame, the temperature profile should result from the coupling between injection, combustion and heat transfer to the gas phase, the dispersed phase and the burner itself. Such a simulation is however beyond the scope of the present study.

Figures 5 and 6 compare the profiles of soot volume fraction and mean particle diameter for increasing temperature levels, $d_{\text {crit }}$ being fixed to its default value, i.e. $13 \mathrm{~nm}$. As discussed in the work of Mueller et al. [13], the soot volume fraction is higher with lower flame temperatures, which is here directly linked to enhanced nucleation. The mean particle diameter also increases following the higher number of particles and the induced multiplication of coalescence events. Coalescence finally stops earlier, hence a ceiling of the particle diameter, already visible at $\phi=2.64$ and $\phi=2.94$, is observed.

\subsubsection{Major gas species}

Figures 9 and 10 present the mole fraction profiles of the major gas species at the three fuel equivalence ratios for the retained value of $d_{c r i t}$, i.e. $18 \mathrm{~nm}$. Fairly good agreement with the measurements is noticed for the main combustion products $\left(\mathrm{CO}, \mathrm{CO}_{2}\right.$ and $\left.\mathrm{H}_{2} \mathrm{O}\right)$ and for the intermediate species $\mathrm{C}_{2} \mathrm{H}_{2}$. The ethylene mole fraction is overestimated downstream the flame front in all cases. However, at the measured levels, experimental uncertainties are known to exceed $15 \%$ [32]. 


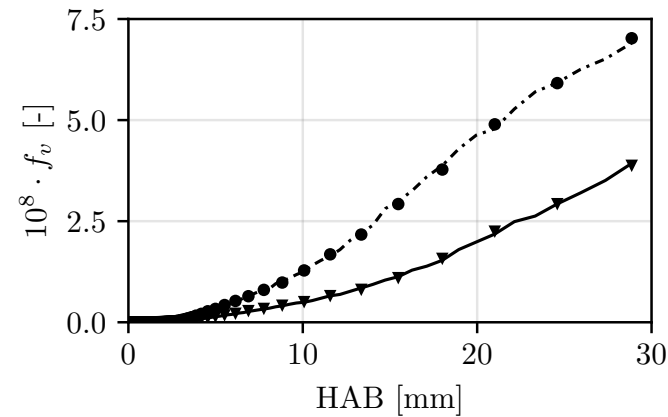

(a) Soot volume fraction

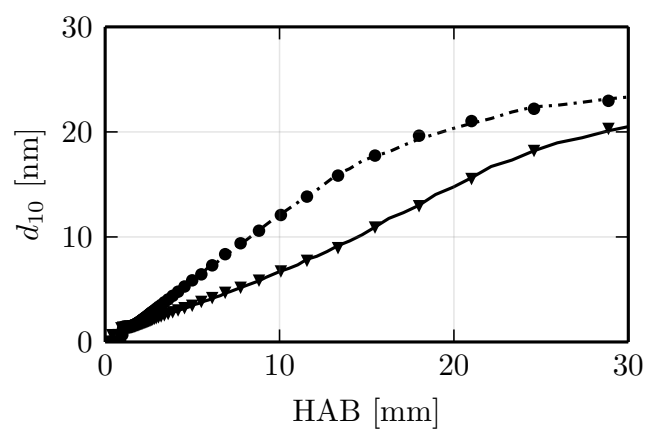

(b) Particle diameter

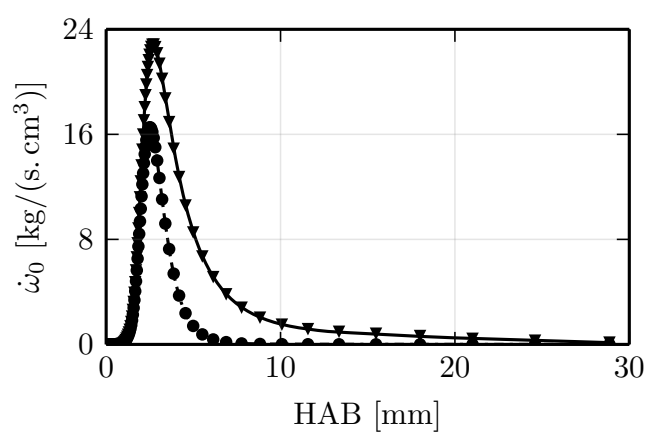

(c) Nucleation rate

Figure 7: Influence of the collision efficiency for condensation: $\gamma_{\mathrm{PAH}} / \gamma_{\mathrm{PAH}^{*}}=0.3 / 1.0(\boldsymbol{\nabla}), 1.0 / 0.3(\mathbf{O}), 0.3 / 0.3$ (-), 1.0/1.0 (-.- ). Variables of interest along height above the burner.

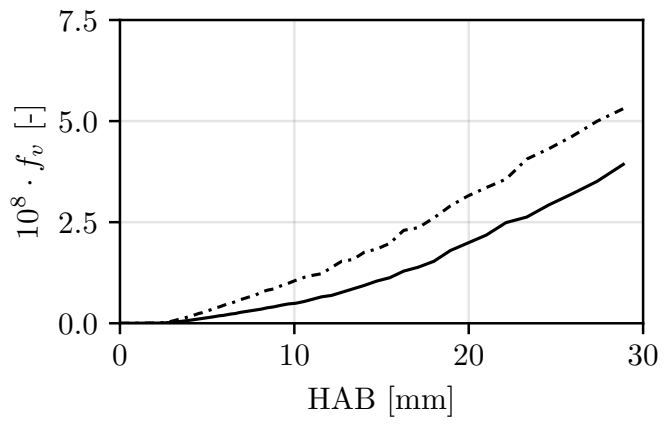

(a) Soot volume fraction

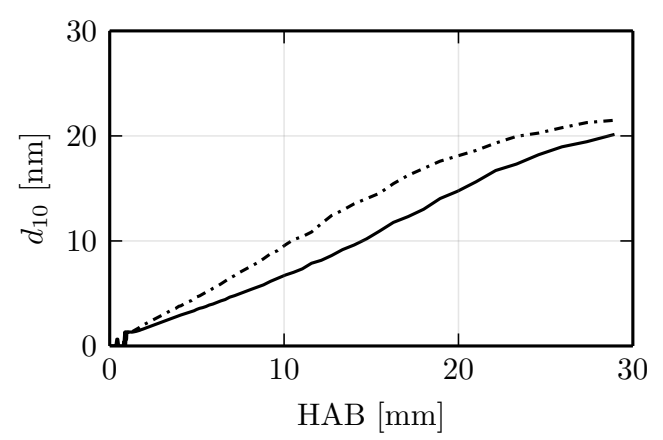

(b) Particle diameter

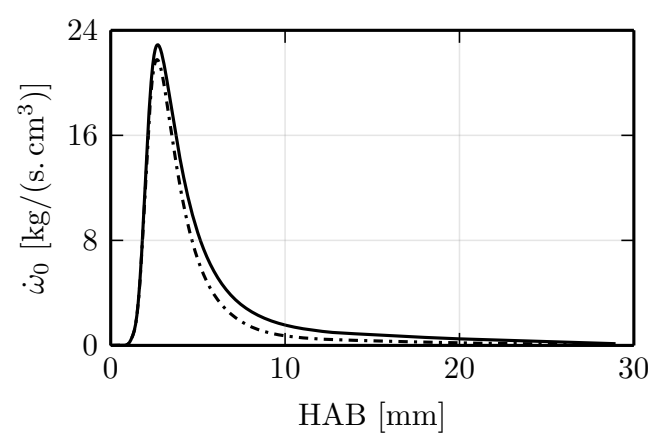

(c) Soot nucleation rate

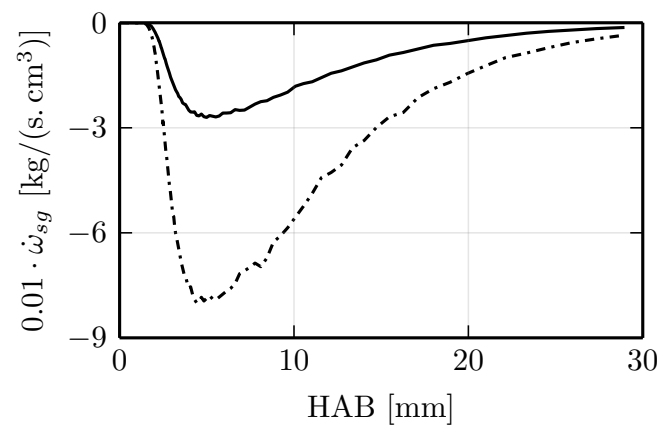

(d) Acetylene mass reaction rate (surface growth)

Figure 8: Influence of the chemical kinetics for $\mathrm{C}_{2} \mathrm{H}_{2}$ addition: $A_{s g}=8.4 \times 10^{5} \mathrm{~m}^{3} /(\mathrm{mol} . \mathrm{s}$ ) (reference, - ), $8.4 \times 10^{6} \mathrm{~m}^{3} /(\mathrm{mol} . \mathrm{s})(-.-)$. Variables of interest along height above the burner. 


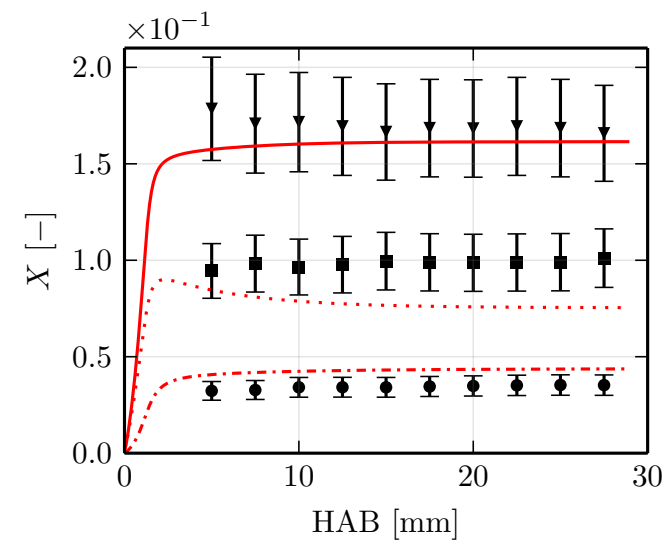

(a) $\phi=2.34$

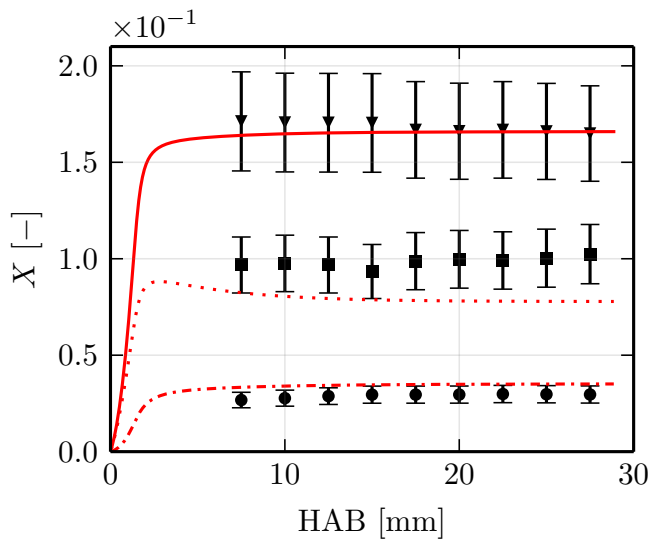

(b) $\phi=2.64$

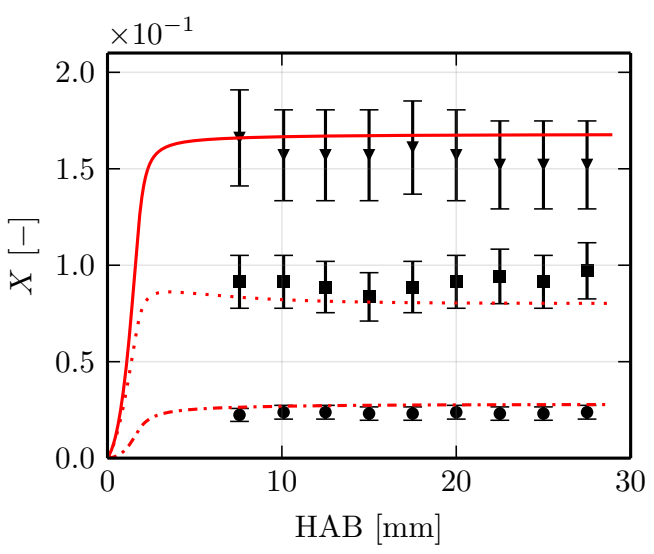

(c) $\phi=2.94$

Figure 9: Mole fractions of the main combustion products: $\mathrm{CO}(-/ \boldsymbol{\nabla}), \mathrm{CO}_{2}(-\cdot-/ \mathbf{0})$ and $\mathrm{H}_{2} \mathrm{O}(\ldots / \mathbf{0})$. Lines are for CEDRE, symbols for experimental measurements.

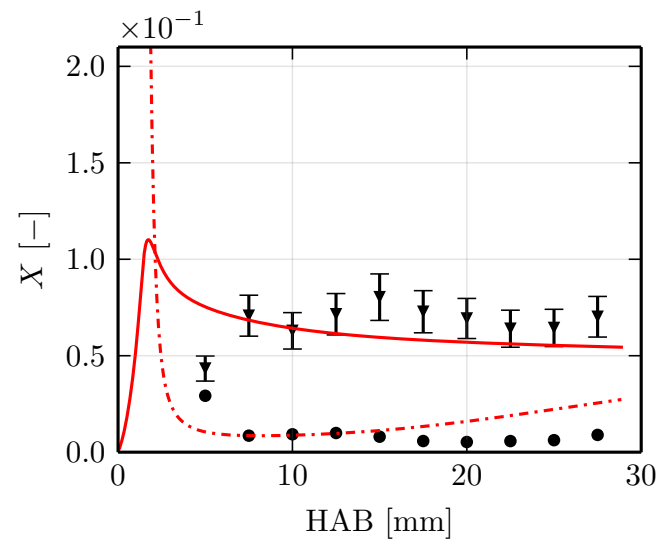

(a) $\phi=2.34$

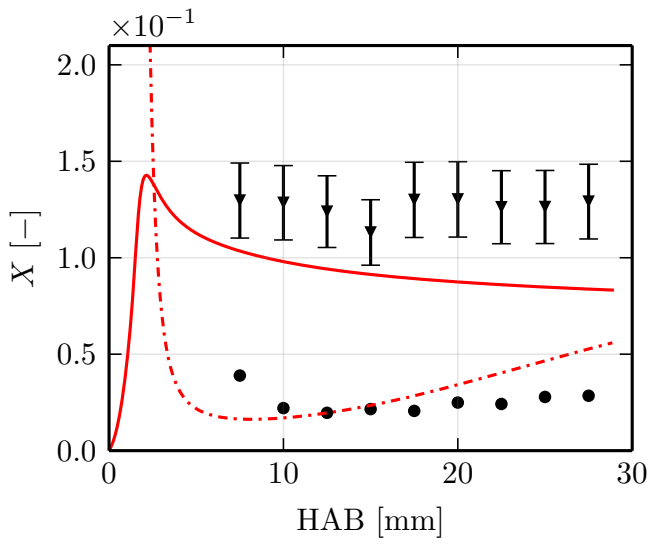

(b) $\phi=2.64$

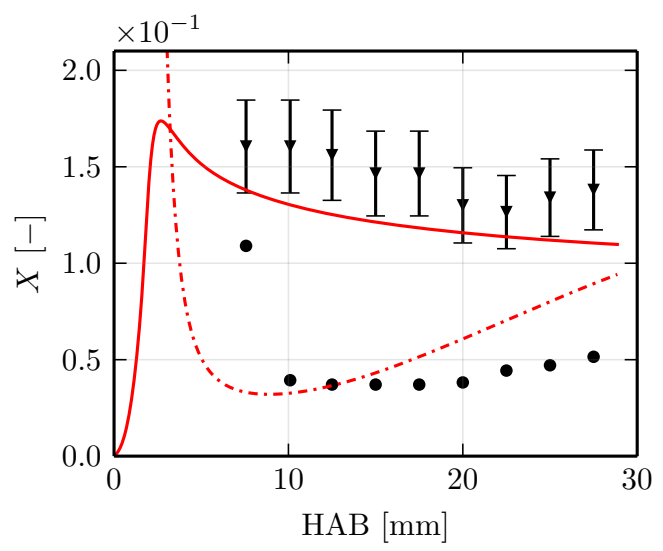

(c) $\phi=2.94$

Figure 10: $\mathrm{C}_{2} \mathrm{H}_{2}$ and $\mathrm{C}_{2} \mathrm{H}_{4}$ mole fractions: $5 \times \mathrm{C}_{2} \mathrm{H}_{2}$ $(-/ \nabla)$ and $50 \times \mathrm{C}_{2} \mathrm{H}_{4}(-\cdot-/ \mathbf{O})$. Lines are for $\mathrm{CE}-$ DRE, symbols for experimental measurements. 


\section{Conclusion}

A Lagrangian description of soot particles evolution and dynamics, coupled with a reduced gas phase reaction mechanism and a sectional model for polycyclic aromatic hydrocarbons, is proposed. It was applied with some success to the simulation of laminar premixed ethylene-air flames at three fuel equivalence ratios. The statistical convergence of the population reduction algorithm - as a function of the reduction intensity and frequency - was first highlighted. The evolution model was then contrasted with experimental measurements, showing its ability to reproduce the growth of the soot volume fraction and the mean particle diameter with increasing equivalence ratio and decreasing temperature. In particular, the suggested description of coalescence processes allows fairly good agreement of the mean particle diameter with the measurements, in terms of both curve shape and level. Finally, the strong sensitivity of soot nucleation and growth to PAH condensation was evidenced.

The present study, restricted to laminar flames, constitutes a prerequisite towards the use of the Lagrangian tracking method in a realistic turbulent combustion chamber. In addition, the modelling of soot aggregates, relevant to radiative heat transfer, has to be addressed, as well as the sensitivity of the critical coalescence diameter over which coalescence is prohibited to the fuel composition and the combustion regime. 


\section{References}

[1] L.-H. Dorey, Modélisation des phénomènes couplés combustion-formation des suies-transferts radiatifs dans les chambres de combustion de turbine à gaz, Ph.D. thesis, École centrale de Paris (2012).

[2] J. B. Moss, Modelling Soot Formation for Turbulent Flame Prediction, Springer Berlin Heidelberg, Berlin, Heidelberg, 1994, pp. 551-568.

[3] B. Magnussen, B. Hjertager, On mathematical modeling of turbulent combustion with special emphasis on soot formation and combustion, Symposium (International) on Combustion 16 (1) (1977) 719-729.

[4] G. Legros, J. Torero, Phenomenological model of soot production inside a non-buoyant laminar diffusion flame, Proceedings of the Combustion Institute 35 (3) (2015) 2545-2553.

[5] K. Leung, R. Lindstedt, W. Jones, A simplified reaction mechanism for soot formation in nonpremixed flames, Combustion and Flame 87 (3) (1991) 289-305.

[6] L. H. Dorey, N. Bertier, L. Tessé, F. Dupoirieux, Soot and radiation modeling in laminar ethylene flames with tabulated detailed chemistry, Comptes Rendus - Mecanique 339 (12) (2011) 756-769.

[7] I. M. Kennedy, Models of soot formation and oxidation, Progress in Energy and Combustion Science 23 (2) (1997) 95-132.

[8] H. Wang, M. Frenklach, A detailed kinetic modeling study of aromatics formation in laminar premixed acetylene and ethylene flames, Combustion and Flame 110 (1-2) (1997) 173-221.

[9] A. D'Anna, J. H. Kent, A model of particulate and species formation applied to laminar, nonpremixed flames for three aliphatic-hydrocarbon fuels, Combustion and Flame 152 (4) (2008) 573-587.

[10] C. Saggese, S. Ferrario, J. Camacho, A. Cuoci, A. Frassoldati, E. Ranzi, H. Wang, T. Faravelli, Kinetic modeling of particle size distribution of soot in a premixed burner-stabilized stagnation ethylene flame, Combustion and Flame 162 (9) (2015) $3356-3369$.

[11] P. Rodrigues, B. Franzelli, R. Vicquelin, O. Gicquel, N. Darabiha, Coupling an LES approach and a soot sectional model for the study of sooting turbulent non-premixed flames, Combustion and Flame 190 (2018) 477-499.

[12] M. Frenklach, H. Wang, Detailed Mechanism and Modeling of Soot Particle Formation, in: H. Bockhorn (Ed.), Soot Formation in Combustion: Mechanisms and Models, Springer Berlin Heidelberg, Berlin, Heidelberg, 1994, pp. 165-192.

[13] M. E. Mueller, G. Blanquart, H. Pitsch, Hybrid Method of Moments for modeling soot formation and growth, Combustion and Flame 156 (6) (2009) 1143-1155.

[14] M. E. Mueller, G. Blanquart, H. Pitsch, A joint volume-surface model of soot aggregation with the method of moments, Proceedings of the Combustion Institute 32 (1) (2009) 785-792.

[15] J. Z. Wen, M. J. Thomson, S. H. Park, S. N. Rogak, M. F. Lightstone, Study of soot growth in a plug flow reactor using a moving sectional model, Proceedings of the Combustion Institute 30 (1) (2005) 1477-1483.

[16] S. H. Park, S. N. Rogak, A novel fixed-sectional model for the formation and growth of aerosol agglomerates, Journal of Aerosol Science 35 (11) (2004) 1385-1404.

[17] C. Eberle, P. M. Gerlinger, M. Aigner, Large Eddy Simulations of a Sooting Lifted Turbulent Jet-Flame, in: 55th AIAA Aerospace Sciences Meeting, AIAA SciTech Forum, American Institute of Aeronautics and Astronautics, 2017.

[18] O. Gicquel, N. Darabiha, D. Thévenin, Laminar premixed hydrogen/air counterflow flame simulations using flame prolongation of ILDM with differential diffusion, Proceedings of the Combustion Institute 28 (2) (2000) 1901-1908.

[19] J. A. Van Oijen, F. A. Lammers, P. H. De Goey, Modeling of complex premixed burner systems by using flamelet-generated manifolds, Combustion and Flame 127 (3) (2001) 2124-2134.

[20] B. Fiorina, R. Vicquelin, P. Auzillon, N. Darabiha, O. Gicquel, D. Veynante, A filtered tabulated chemistry model for LES of premixed combustion, Combustion and Flame 157 (3) (2010) 465-475.

[21] A. Boucher, N. Bertier, F. Dupoirieux, A method to extend flamelet manifolds for prediction of NOx and long time scale species with tabulated chemistry, International Journal of Sustainable Aviation 1 (2) (2014) 181-202.

[22] B. Zamuner, F. Dupoirieux, Numerical Simulation of Soot Formation in a Turbulent Flame with a Monte-Carlo PDF Approach and Detailed Chemistry, Combustion Science and Technology 158 (1) (2000) 407-438.

[23] H. Richter, S. Granata, W. H. Green, J. B. Howard, Detailed modeling of PAH and soot formation in a laminar premixed benzene/oxygen/argon low-pressure flame, Proceedings of the Combustion Institute 30 (1) (2005) 1397-1405.

[24] M. Di Domenico, P. Gerlinger, M. Aigner, Development and validation of a new soot formation model for gas turbine combustor simulations, Combustion and Flame 157 (2) (2010) 246-258.

[25] T. Blacha, M. Di Domenico, P. Gerlinger, M. Aigner, Soot predictions in premixed and non-premixed laminar flames using a sectional approach for PAHs and soot, Combustion and Flame 159 (1) (2012) 181-193.

[26] Lagrangian tracking of soot particles in LES of gas turbines, Proceedings of the Combustion Institute 37 (4) (2018) 5429-5436.

[27] J. C. Ong, K. M. Pang, J. H. Walther, J.-H. Ho, H. K. Ng, Evaluation of a Lagrangian Soot Tracking Method for the prediction of primary soot particle size under engine-like conditions, Journal of Aerosol Science 115 (2018) 70-95.

[28] A. Refloch, B. Courbet, A. Murrone, P. Villedieu, C. Laurent, P. Gilbank, J. Troyes, L. Tessé, G. Chaineray, J. Dargaud, et al., Cedre software, AerospaceLab (2) (2011).

[29] J.-L. Consalvi, F. Liu, J. Contreras, M. Kashif, G. Legros, S. Shuai, J. Wang, Numerical study of soot formation in laminar coflow diffusion flames of methane doped with primary reference fuels, Combustion and Flame 162 (4) (2015) 1153-1163.

[30] J. Hirschfelder, R. B. Bird, C. F. Curtiss, Molecular theory of gases and liquids (1964).

[31] T. Poinsot, D. Veynante, Theoretical and Numerical Combustion, 2nd Edition, R.T. Edwards, Inc., 2005.

[32] F. Xu, P. B. Sunderland, G. M. Faeth, Soot formation in laminar premixed ethylene/air flames at atmospheric pressure, Combustion and Flame 108 (4) (1997) 471-493. 
[33] R. A. Dobbins, Soot inception temperature and the carbonization rate of precursor particles, Combustion and Flame 130 (3) (2002) 204-214.

[34] F. A. Williams, Spray Combustion and Atomization, Physics of Fluids 1 (6) (1958) 541-545.

[35] P. Villedieu, J. Hylkema, Une méthode particulaire aléatoire reposant sur une équation cinétique pour la simulation numérique des sprays denses de gouttelettes liquides, Comptes Rendus de l'Académie des Sciences - Series I - Mathematics 325 (3) (1997) 323-328.

[36] J. Hylkema, P. Villedieu, A random particle method to simulate coalescence phenomena in dense liquid sprays, in: C.-H. Bruneau (Ed.), Sixteenth International Conference on Numerical Methods in Fluid Dynamics, Springer Berlin Heidelberg, Berlin, Heidelberg, 1998, pp. 488-493.

[37] J. J. Hylkema, Modélisation cinétique et simulation numérique d'un brouillard dense de gouttelettes : application aux propulseurs à poudre, Ph.D. thesis (1999)

[38] M. De Berg, O. Cheong, M. Van Kreveld, M. Overmars, Computational Geometry: Algorithms and Applications, in: Computational Geometry, 2008, pp. 307-322.

[39] C. Eberle, P. Gerlinger, M. Aigner, A sectional PAH model with reversible PAH chemistry for CFD soot simulations, Combustion and Flame 179 (2017) 63-73.

[40] C. J. Pope, J. B. Howard, Simultaneous Particle and Molecule Modeling (SPAMM): An Approach for Combining Sectional Aerosol Equations and Elementary Gas-Phase Reactions, Aerosol Science and Technology 27 (1) (1997) $73-94$.

[41] A. Murrone, P. Villedieu, Numerical modeling of dispersed two-phase flows, AerospaceLab (2) (2011).

[42] M. Frenklach, H. Wang, Detailed modeling of soot particle nucleation and growth, Symposium (International) on Combustion 23 (1) (1991) 1559-1566.

[43] A. Kazakov, H. Wang, M. Frenklach, Detailed modeling of soot formation in laminar premixed ethylene flames at a pressure of 10 bar, Combustion and Flame 100 (1-2) (1995) 111-120.

[44] K. G. Neoh, J. B. Howard, A. F. Sarofim, Soot Oxidation in Flames, in: D. C. Siegla, G. W. Smith (Eds.), Particulate Carbon: Formation During Combustion, Springer US, Boston, MA, 1981, pp. 261-282.

[45] S. J. Harris, I. M. Kennedy, The Coagulation of Soot Particles with van der Waals Forces, Combustion Science and Technology 59 (4-6) (1988) 443-454.

[46] N. A. Fuchs, The mechanics of aerosols, Pergamon Press, Oxford, 1964

[47] International Sooting Flame (ISF) Workshop. URL https://www . adelaide.edu.au/cet/isf workshop/data-sets

[48] D. G. Goodwin, H. K. Moffat, R. L. Speth, Cantera: An object-oriented software toolkit for chemical kinetics, thermodynamics, and transport processes, http://www.cantera.org, version 2.3.0 (2017). 


\section{Declaration of interests}

$\bigotimes$ The authors declare that they have no known competing financial interests or personal relationships that could have appeared to influence the work reported in this paper.

$\square$ The authors declare the following financial interests/personal relationships which may be considered as potential competing interests: 